\title{
ESTABILIDADE DE MELÃO PROCESSADO POR DESIDRATAÇÃO OSMÓTICA SEGUIDA DE FRITURA
}

\author{
Stability of melon processed by osmotic dehydration followed by frying
}

\author{
Janice Ribeiro Lima', Laura Maria Bruno²
}

\begin{abstract}
RESUMO
A desidratação osmótica seguida de fritura tem se apresentado como uma ferramenta tecnológica importante no desenvolvimento de novos produtos. Com o presente trabalho, visou-se avaliar a estabilidade de melão obtido por desidratação osmótica e fritura. A estabilidade do produto foi avaliada segundo suas características físico-químicas (sólidos solúveis totais, atividade de água, pH, umidade, acidez, açúcares redutores e não-redutores), microbiológicas (coliformes totais e fecais, Escherichia coli, Salmonella sp., Staphylococcus coagulase positiva, aeróbios mesófilos, bolores e leveduras) e sensoriais (aceitação), durante 124 dias de armazenamento à temperatura ambiente $\left(\sim 28^{\circ} \mathrm{C}\right)$. A atividade de água e a umidade apresentaram tendência de aumento durante o armazenamento, enquanto que os demais parâmetros físico-químicos, apesar de terem algumas oscilações, ficaram praticamente constantes. A aceitação sensorial também foi constante, sendo a avaliação próxima de gostei moderadamente. As análises microbiológicas demonstraram boa qualidade do produto durante todo o armazenamento, estando dentro dos padrões exigidos pela legislação brasileira: ausência de Salmonella sp. em $25 \mathrm{~g}$ e máximo de $10^{2} \mathrm{NMP} / \mathrm{g}$ de coliformes fecais $\left(45^{\circ} \mathrm{C}\right)$.
\end{abstract}

Termos para indexação: Cucumis melo L., processamento, armazenamento.

\begin{abstract}
Osmotic dehydration followed by frying has been considered as an important technological tool to the development of new products. The objective of the present work was to evaluate storage stability of melon processed by osmotic dehydration and frying. The stability was assessed by physical-chemical (total soluble solids, water activity, $\mathrm{pH}$, moisture content, acidity, reducing and non reducing sugars), microbiological (total and fecal coliforms, Escherichia coli, Salmonella sp., Staphylococcus, plate count, east and mold) and sensory methods (acceptance), during 124 days of storage at room temperature $\left(\sim 28^{\circ} \mathrm{C}\right)$. Water activity and moisture content increased during storage while others physical chemicals parameters remained constant. Sensory acceptance was constant and evaluation was near the note like moderately. Microbiological analysis showed good product quality during the storage, being within Brazilian legislation: Salmonella sp. absence in $25 \mathrm{~g}$ and maximum of $10^{2} \mathrm{MPN} / \mathrm{g}$ of fecal coliforms $\left(45^{\circ} \mathrm{C}\right)$.
\end{abstract}

Index terms: Cucumis melo L., processing, storage.

(Recebido para publicação 10 de janeiro de 2006 e aprovado em 25 de maio de 2006)

\section{INTRODUÇÃO}

A demanda por produtos naturais, saudáveis e à base de frutas tem crescido muito, não apenas como produtos acabados, mas também como ingredientes a serem incluídos em alimentos mais elaborados, como sorvetes, cereais, laticínios, produtos de confeitaria e panificação. Nesse contexto, o tratamento osmótico tem se apresentado como uma ferramenta tecnológica importante para se desenvolver novos produtos derivados de frutas (TORREGGIANI \& BERTOLO, 2001).

Tratamentos osmóticos são usados principalmente como pré-tratamento introduzido em alguns processos convencionais, tais como secagem a ar quente, fritura, microondas e liofilização, a fim de melhorar a qualidade do produto final, reduzir custos de energia ou mesmo formular novos produtos (SERENO et al., 2001). Segundo Maestrelli et al. (2001), o uso da desidratação osmótica, combinada ou não a outros processos, tem sido efetivo na redução do colapso estrutural de frutos delicados, mesmo quando posteriormente se aplicam processos agressivos como tratamento térmico (KROKIDA et al., 2000).

A desidratação osmótica permite tanto a remoção de água do produto quanto a modificação de suas propriedades pela impregnação de solutos desejados (MIZRAHI et al., 2001). Essa técnica emprega soluções de alta pressão osmótica, em que dois fluxos são estabelecidos: a saída de água do alimento para a solução e a incorporação do soluto pelo alimento, devido aos gradientes de concentração (TORREGGIANI, 1993).

O processo de fritura, utilizado como complemento da osmose, além de reduzir a atividade de água por evaporação da água presente, reduz a carga microbiana, pelo efeito do calor, favorecendo a qualidade dos produtos obtidos.

Com o presente trabalho, visou-se avaliar a estabilidade de melão desidratado obtido por desidratação osmótica seguida de fritura.

'Engenheira de Alimentos - Embrapa Agroindústria Tropical - Rua Dra. Sara Mesquita, 2270, Planalto Pici - 60511-110 - Fortaleza, CE janice@cnpat.embrapa.br

${ }^{2}$ Engenheira de Alimentos - Embrapa Agroindústria Tropical - Rua Dra. Sara Mesquita, 2270, Planalto Pici - 60511-110 - Fortaleza, CE Imbruno@cnpat.embrapa.br 


\section{MATERIALE MÉTODOS}

As condições de processamento do melão foram baseadas nos resultados obtidos por Lima et al. (2004) e Silva et al. (2004). Os frutos (Cucumis melo L. c.v. Cantaloupe) foram adquiridos no mercado local, em Fortaleza, Estado do Ceará, selecionados, lavados em água clorada $(50 \mathrm{ppm} / 15 \mathrm{~min})$, descascados e cortados em cubos.

O tratamento osmótico foi realizado com xarope de sacarose a $65^{\circ} \mathrm{Brix}$, na proporção fruto:xarope de $1: 2$, a $65^{\circ} \mathrm{C}$ por $5 \mathrm{~h}$. A solução osmótica continha $0,1 \%$ de benzoato de sódio e ácido cítrico (q.s.p. pH 3,0). Após o processo osmótico, os frutos foram fritos por imersão em gordura vegetal hidrogenada, a $140^{\circ} \mathrm{C}$ por 8 minutos. Após o processamento, o produto foi acondicionado em embalagens rígidas de polipropileno, com capacidade de $200 \mathrm{~g}$ cada uma e armazenado à temperatura ambiente $\left(\sim 28^{\circ} \mathrm{C}\right)$ por 124 dias.

Avaliações do produto foram realizadas a $0,32,62$, 92 e 124 dias. A cada período de análise foram retiradas três embalagens, sendo de cada uma delas retiradas $25 \mathrm{~g}$ de amostras que foram homogeneizadas para realização dos testes microbiológicos. O conteúdo restante foi homogeneizado, obtendo-se uma amostra composta para as análises físico-químicas, que foram realizadas em triplicata.
As seguintes análises físico-químicas foram realizadas: $\mathrm{pH}$, acidez, sólidos solúveis totais e umidade (INSTITUTO ADOLFO LUTZ, 1985), açúcares redutores e não-redutores (MILLER, 1959) e atividade de água (aparelho Decagon CX-2). O teor de gordura foi determinado na amostra inicial (CECCHI, 1999).

As análises microbiológicas de aeróbios mesófilos, coliformes fecais e totais, Escherichia coli, bolores e leveduras, Salmonella sp. e Staphylococcus coagulase positiva foram realizadas segundo a metodologia descrita no Compendium of Methods for the Microbiological Examination of Foods (DOWES \& ITO, 2001).

Para os testes sensoriais, cinco embalagens foram utilizadas a cada período de análise, sendo que os frutos contidos em todas as embalagens foram misturados para serem servidos aos provadores. Utilizou-se escala hedônica estruturada de 9 pontos, variando de desgostei muitíssimo (nota 1) a gostei muitíssimo (nota 9) (MEILGAARD et al., 1987). As amostras foram apresentadas aos provadores, em copos plásticos brancos. Foram utilizados 35 provadores não treinados, que avaliaram as amostras quanto à aceitação global.

Os resultados das análises físico-químicas e sensoriais foram avaliados por análise de variância e teste de média (Tukey) a 5\% de significância.

O fluxograma da Figura 1 ilustra o processamento do melão.

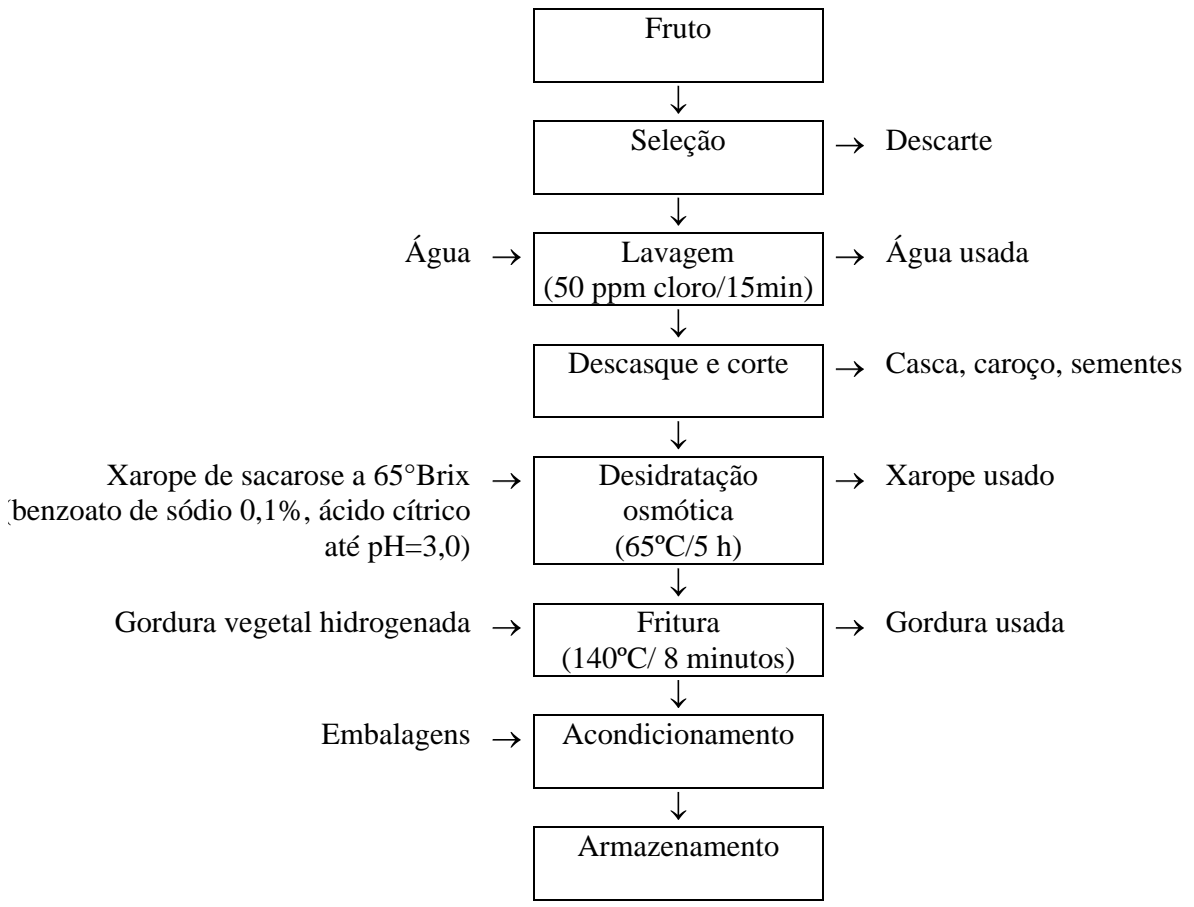

FIGURA 1 - Fluxograma geral do processamento de melão.

Ciênc. agrotec., Lavras, v. 30, n. 5, p. 937-941, set./out., 2006 


\section{RESULTADOS E DISCUSSÃO}

Os resultados do teste de estabilidade são mostrados na Tabela 1.

$\mathrm{O}$ teor de gordura foi determinado apenas na amostra inicial. $\mathrm{O}$ valor observado $(2,3 \%)$ foi pequeno, apesar da porosidade do fruto, provavelmente como resultado do pré-tratamento osmótico. Querido (2005) observa que no emprego de fritura de frutas é importante garantir baixo teor de gordura e que a utilização de osmose, como pré-tratamento, reduz a absorção de gordura durante a fritura.

Os relatos encontrados na literatura sobre o processamento de frutos por fritura, visam a produção de chips, um produto com umidade muito inferior e maior teor de gordura do que o obtido nesse experimento, o que torna difícil a comparação de resultados. Reynes \& Aymard (1997), citado por Querido (2005) em estudo de produção de chips de abacaxi por desidratação osmótica e fritura obtiveram incorporação de óleo mínima de 14,02 a 16,87\%. European Patent Application (1989) apresenta um processo para produção de chips de frutas e hortaliças envolvendo as etapas de corte, secagem osmótica (solução de sacarose $30 \mathrm{~g} / 100 \mathrm{~g}$ ), fritura sob vácuo a 80$130^{\circ} \mathrm{C}$ por $0,5-4,0$ minutos. Os produtos resultantes apresentaram umidade máxima de $4 \mathrm{~g} / 100 \mathrm{~g}$ e teor de gordura de $15-30 \mathrm{~g} / 100 \mathrm{~g}$.

Pela Tabela 1, observa-se que a umidade e a atividade de água apresentaram tendência de aumento. Trocas gasosas com o meio e diferenças de umidade relativa ao longo do ano devem ser responsáveis por essas diferenças, já que a embalagem de polipropileno, material utilizado nesse experimento, não é de alta barreira à umidade (GARCIA et al., 1989).

Pouca alteração foi observada nos sólidos solúveis durante o armazenamento. $\mathrm{O}$ pH ficou entre 4,47 e 4,73, o que poderia ser um fator preocupante com relação aos perigos biológicos, no entanto, a atividade de água reduzida e o uso de benzoato de sódio são fatores coadjuvantes na manutenção da qualidade do produto.

A acidez sofreu pequenas oscilações ao longo do período de armazenamento, ficando abaixo de $0,50 \%$ para todos os tempos.
Apesar da presença de ácidos no meio, o que tenderia a provocar a hidrólise da sacarose, os valores de açúcares não-redutores e de açúcares redutores não apresentaram alteração, não se considerando o valor mais alto observado no tempo de 62 dias, provavelmente devido a problemas de amostragem.

A aceitação sensorial foi alta, não apresentando queda durante o armazenamento. Os valores observados correspondem a uma avaliação de gostei moderadamente e ficaram na faixa de aceitação, que engloba todas as notas variando de 6 a 9 .

Dentre os relatos encontrados na literatura sobre fritura de frutas, a produção de chips de banana é um dos mais usuais. Akubor \& Adejo (2000) avaliaram a estabilidade de chips de banana produzidos por fritura em imersão em óleo de amendoim. Os chips foram embalados em sacos de polietileno e armazenados a $30^{\circ} \mathrm{C}$ por 3 meses. Os autores não observaram redução das notas sensoriais até 2 meses de armazenamento, mas após 3 meses, diferenças foram observadas.

As análises microbiológicas demonstraram boa qualidade do produto, estando dentro dos padrões exigidos pela legislação brasileira, ou seja, ausência de Salmonella sp. em 25 g e máximo de $10^{2} \mathrm{NMP} / \mathrm{g}$ de coliformes fecais (ANVISA, 2001) (Tabela 2). Também não foram observadas contagens de Escherichia coli e Staphylococcus sp. Esses resultados são um reflexo das boas condições de higiene empregadas na elaboração do produto e das características físico-químicas obtidas (baixa atividade de água, baixo $\mathrm{pH}$ em associação ao benzoato de sódio).

A contagem de bolores e leveduras observada no início e ao final do armazenamento indicou que ocorreu contaminação do produto após a fritura, visto que a temperatura $\left(140{ }^{\circ} \mathrm{C}\right)$ utilizada no processo promove destruição dos microrganismos presentes. Da mesma forma, foram observadas contagens de microrganismos aeróbios mesófilos a 92 dias de armazenamento. Essas contaminações foram pontuais, já que o mesmo comportamento não se repetiu nos tempos subseqüentes de análise. Vale ressaltar que, a população de microrganismos presentes (inferior a $10^{5} \mathrm{UFC} / \mathrm{g}$ ) nas amostras analisadas não comprometeu a qualidade do produto, visto que as características sensoriais das amêndoas não foram afetadas. 
TABELA 1 - Características de melão desidratado por osmose e fritura durante armazenamento (média \pm desvio padrão).

\begin{tabular}{|c|c|c|c|c|c|}
\hline \multirow{2}{*}{ Análises } & \multicolumn{5}{|c|}{ Tempo de armazenamento (dias) } \\
\hline & $\mathbf{0}$ & 32 & 62 & 92 & 124 \\
\hline Sólidos solúveis $\left({ }^{\circ} \mathrm{Brix}\right)$ & $64,13 \pm 0,94 b$ & $68,03 \pm 0,35 \mathrm{a}$ & $68,10 \pm 0,79 \mathrm{a}$ & $62,50 \pm 2,10 \mathrm{~b}$ & $69,70 \pm 1,99 \mathrm{a}$ \\
\hline Atividade de água & $0,77 \pm 0,01 \mathrm{c}$ & $0,72 \pm 0,01 \mathrm{~d}$ & $0,80 \pm 0,01 \mathrm{ab}$ & $0,79 \pm 0,01 \mathrm{~b}$ & $0,81 \pm 0,01 \mathrm{a}$ \\
\hline $\mathrm{pH}$ & $4,73 \pm 0,06 \mathrm{a}$ & $4,57 \pm 0,06 \mathrm{~b}$ & $4,47 \pm 0,06 \mathrm{~b}$ & $4,60 \pm 0,01 \mathrm{ab}$ & $4,47 \pm 0,06 \mathrm{~b}$ \\
\hline Umidade $(\%)$ & $19,60 \pm 0,56 \mathrm{~d}$ & $22,53 \pm 0,40 \mathrm{c}$ & $18,67 \pm 0,59 \mathrm{~d}$ & $25,93 \pm 0,76 b$ & $28,70 \pm 0,10 \mathrm{a}$ \\
\hline Acidez (g ac. cítrico/100 g) & $0,38 \pm 0,01 \mathrm{~b}$ & $0,24 \pm 0,01 \mathrm{c}$ & $0,46 \pm 0,05 \mathrm{a}$ & $0,24 \pm 0,01 \mathrm{c}$ & $0,30 \pm 0,01 \mathrm{c}$ \\
\hline Açúcares redutores (\%) & $39,60 \pm 1,56 b$ & $38,5 \pm 0,14 b$ & $44,80 \pm 1,69 \mathrm{a}$ & $36,90 \pm 0,28 b$ & $39,70 \pm 1,69 b$ \\
\hline Açúcares não-redutores (\%) & $24,25 \pm 0,92 \mathrm{a}$ & $23,30 \pm 1,84 \mathrm{a}$ & $23,15 \pm 1,46$ a & $26,75 \pm 0,07 \mathrm{a}$ & $28,65 \pm 1,48$ a \\
\hline $\begin{array}{l}\text { Aceitação sensorial } \\
\text { (escala de } 9 \text { pontos) }\end{array}$ & $6,82 \pm 1,97 \mathrm{a}$ & $6,05 \pm 2,02 \mathrm{a}$ & $\mathrm{Nd}$ & $6,80 \pm 1,66 \mathrm{a}$ & $7,03 \pm 1,67 \mathrm{a}$ \\
\hline Teor de gordura $(\%)$ & 2,3 & $\mathrm{Nd}$ & $\mathrm{Nd}$ & $\mathrm{Nd}$ & $\mathrm{Nd}$ \\
\hline
\end{tabular}

Nd- não determinado. Em cada linha, amostras seguidas de mesmas letras não diferem significativamente a 5\% pelo teste de Tukey.

TABELA 2 - Características microbiológicas de melão desidratado por osmose e fritura durante armazenamento.

\begin{tabular}{|c|c|c|c|c|c|c|c|}
\hline $\begin{array}{l}\text { Tempo } \\
\text { (dias) }\end{array}$ & $\begin{array}{l}\text { Coliformes } \\
\text { totais } \\
(\mathrm{NMP} / \mathrm{g}) \\
\end{array}$ & $\begin{array}{l}\text { Coliformes } \\
\text { fecais } \\
(\mathrm{NMP} / \mathrm{g}) \\
\end{array}$ & $\begin{array}{c}\text { Escherichia } \\
\text { coli } \\
(\mathrm{NMP} / \mathrm{g})\end{array}$ & $\begin{array}{c}\text { Salmonella } \\
\text { sp. } \\
(\mathrm{em} 25 \mathrm{~g})\end{array}$ & $\begin{array}{c}\text { Staphylococcu } \\
\text { s sp. } \\
\text { (UFC/g) }\end{array}$ & $\begin{array}{c}\text { Aeróbios } \\
\text { mesófilos } \\
\text { (UFC/g) }\end{array}$ & $\begin{array}{c}\text { Bolores e } \\
\text { leveduras } \\
\text { (UFC/g) }\end{array}$ \\
\hline 0 & $<3$ & $<3$ & $<3$ & ausência & $<100$ & $<10$ & $7,8 \times 10^{3}$ \\
\hline 32 & $<3$ & $<3$ & $<3$ & ausência & $<100$ & $<10$ & $<100$ \\
\hline 62 & 9 & 4 & $<3$ & ausência & $<100$ & $<10$ & $<100$ \\
\hline 92 & $<3$ & $<3$ & $<3$ & ausência & $<100$ & $2,1 \times 10^{4}$ & $<100$ \\
\hline 124 & $<3$ & $<3$ & $<3$ & ausência & $<100$ & $<10$ & $1,9 \times 10^{2}$ \\
\hline
\end{tabular}

\section{CONCLUSÃO}

Não houve redução significativa da aceitação sensorial do melão processado ao longo do período de armazenamento e este ainda se encontrava dentro dos padrões microbiológicos exigidos pela legislação brasileira ao final de quatro meses. Portanto, pode-se concluir que o emprego de desidratação osmótica seguida de fritura permite a elaboração de melão desidratado, obtendo-se um produto estável à temperatura ambiente, durante pelo menos quatro meses.

\section{REFERÊNCIAS BILIOGRÁFICAS}

AGÊNCIA NACIONAL DE VIGILÂNCIA SANITÁRIA. Resolução RDC n. 12, de 2 de janeiro de 2001. Regulamento técnico sobre os padrões microbiológicos para alimentos. Diário Oficial da União, Brasília, p. 1-54, jan. 2001.
AKUBOR, P. I.; ADEJO, E. E. Physicochemical, microbiological and sensory changes in stored plantain chips. Plant Foods for Human Nutrition, Netherlands, v. 55, n. 2, p. 139-146, 2000.

CECCHI, H. M. Fundamentos teóricos e práticos em análise de alimentos. Campinas: Unicamp, 1999. 212 p.

DOWNES, F. P.; ITO, K. Compendium of methods for the microbiological examination of foods. 4. ed. Washington, DC: American Public Health Association, $2001.676 \mathrm{p}$.

EUROPEAN PATENT APPLICATION. P. P. Tobback; M. C. Feys. Process for production of fruit or vegetable chips. EP 0339175 A1, 1989. 
GARCIA, E. E. C.; PADULA, M.; SARANTOPOULOS, C. I. G. L. Embalagens plásticas: propriedades de barreira. Campinas: Instituto de Tecnologia de Alimentos, 1989. 44 p.

INSTITUTO ADOLFO LUTZ. Normas analíticas do Instituto Adolfo Lutz: métodos químicos e físicos para análises de alimentos. 3. ed. São Paulo, 1985. v. 1, 533 p.

KROKIDA, M. K.; KARATHANOS, V. T.; MAROULIS, Z. B.; MARINOS-KOURIS, D. Effect of osmotic dehydration on color and sorption characteristics of apple and banana. Drying Technology, New York, v. 18, n. 6, p. 937-950, 2000.

LIMA, A. S.; FIGUEIREDO, R. W.; MAIA, G. A.; LIMA, J. R.; SOUZA NETO, M. A.; SOUZA, A. C. R. Estudo das variáveis de processo sobre a cinética de desidratação osmótica de melão. Ciência e Tecnologia de Alimentos, Campinas, v. 24, n. 2, p. 282-286, 2004.

MAESTRELLI, A.; SCALZO, R.; LUPI, D.; BERTOLO, G.; TORREGGIANI, D. Partial removal of water before freezing: cultivar and pre-treatments as quality factors of frozen muskmelon (Cucumis melo, cv. reticulatus Naud). Journal of Food Engineering, Oxford, v. 49, n. 2, p. 255-260, 2001.

MEILGAARD, M.; CIVILLE, G. V.; CARR, B. T. Sensory evaluation techniques. Florida: CRC, 1987. v. 2, 158 p.

MILLER, G. L. Use of dinitrosalicycle acid reagent for determination of reducing sugars. Analytical Chemistry, Washington, n. 31, p. 226-248, 1959.
MIZRAHI, S.; EICHLER, S.; RAMON, O. Osmotic dehydration phenomena in gel systems. Journal of Food Engineering, Oxford, v. 49, n. 1, p. 87-96, 2001.

QUERIDO, A. F. Estudo da influência de técnicas na minimização da incorporação de óleo durante o processo de fritura de maçã. 2005. 219 f. Tese (Doutorado em Tecnologia de Alimentos) - Universidade Estadual de Campinas, Campinas, 2005.

SERENO, A. M.; HUBINGER, M. D.; COMESAÑA, J. F.; CORREA, A. Prediction of water activity of osmotic solutions. Journal of Food Engineering, Oxford, v. 49, n. 1, p. 103-114, 2001.

SILVA, V. K. L.; OLIVEIRA, M. A.; LIMA, J. R. Efeito do tempo e temperatura de fritura na atividade de água de melão desidratado por osmose/fritura. In: CONGRESSO BRASILEIRO DE CIÊNCIA E TECNOLOGIA DE ALIMENTOS, 19., 2004, Recife. Anais... Recife: SBCTA, 2004. CD-ROM.

TORREGGIANI, D. Osmotic dehydration in fruit and vegetable processing. Food Research International, Monticello, v. 26, n. 1, p. 59-68, 1993.

TORREGGIANI, D.; BERTOLO, G. Osmotic pre-treatments in fruit processing: chemical, physical and structural effects. Journal of Food Engineering, Oxford, v. 49, n. 2, p. 247-253, 2001. 